Bull. Austral. Math. Soc.

$05 \mathrm{C} 15,05 \mathrm{C} 55$

VOL. 37 (1988) [197-212]

\title{
MONOCHROMATIC TRIANGLES IN THREE COLOURS
}

\author{
S.S. SANE AND W.D. Wallis
}

\begin{abstract}
Suppose the edges of the complete graph on 17 vertices are coloured in three colours. It is shown that at least five monochromatic triangles must arise.
\end{abstract}

\section{INTRODUCTION}

By a proper colouring of a graph $G$ in $n$ colours is meant a way of assigning $n$ or fewer colours to the edges of $G$ in such a way that no monochromatic triangle results.

We are interested in edge-colourings of complete graphs. If $K_{r}$ denotes the complete graph on $r$ vertices, then Ramsey's theorem guarantees the existence of a number $r_{n}(2)$ such that $K_{r}$ has a proper colouring in $n$ colours if and only if $r<r_{n}(2)$. (For a discussion see [7].) These numbers have been investigated; however only $r_{2}(2)$ and $r_{3}(2)$ are known.

Proposition 1. [6] $r_{2}(2)=6 ; r_{3}(2)=17$.

Since $r_{2}(2)=6$, any colouring of $K_{6}$ must contain a monochromatic triangle. In fact, it can be shown that at least two triangles must occur. This follows from a more general result of Goodman:

Proposition 2. [1] In any edge-colouring of $K_{r}$ in two colours there must be at least $f(r)$ monochromatic triangles, where:

$$
\begin{aligned}
& f(r)=\frac{1}{24} r(r-2)(r-4) \text { if } r \text { is even; } \\
& f(r)=\frac{1}{24} r(r-1)(r-5) \text { if } r \equiv 1(\bmod 4) ; \\
& f(r)=\frac{1}{24}(r+1)(r-3)(r-4) \text { if } r \equiv 3(\bmod 4) .
\end{aligned}
$$

Moreover, these bounds can all be attained.

When the number of colours is 3 , there must clearly be a function like $f$, but we know very little about it.- A recent paper by Goodman [2] discusses the 3 -colour

Received 6 May 1987

Both authors aknowledge the hospitality of the University of Waterloo where some of this research was carried out. The work of the second author was partially supported by NSF grant DMS 8601828.

Copyright Clearance Centre, Inc. Serial-fee code: 0004-9729/88 \$A2.00+0.00. 
case, and earlier we showed in $[6]$ that the number of monochromatic triangles in a 3 -colouring of the edges of $K_{17}$ must be at least 3 , and that 5 could be attained. But the main information to be gleaned from those two papers is that this problem is very much more difficult than the 2-colour case.

Our object in this paper is to prove the following result, which was conjectured in $[\boldsymbol{6}]$.

Proposition 3. At least five monochromatic triangles occur in any 3-colouring of the edges of $K_{17}$.

Harary and Prins [5] define the Ramsey multiplicity of a graph. In their terminology, the result of Goodman for $r=6$ can be expressed by saying that the Ramsey multiplicity of $K_{3}$ is 2, and one might say that we shall prove the "Ramsey 3-colour multiplicity" of $K_{3}$ to be 5 . For results on Ramsey multiplicities of other graphs, see $[\mathbf{5}]$.

A related result of interest is the proof by Harary [4] that up to isomorphism there are precicely seven ways of edge-colouring $K_{6}$ in two colours with precisely two monochromatic triangles. We do not need all of that paper, but we shall use some results on the structure of colourings with only two monochromatic triangles. For convenience we prove them in the next section, but we point out that the proofs can be found by going through [4].

\section{Some terminology}

In most of this paper we are concerned with colouring $K_{17}$. We assume that the colours are red, blue and green. If $x$ is any vertex, then $R(x), B(x)$ and $G(x)$ are the sets of vertices joined to $x$ by red, blue and green edges respectively; $r(x)$ is the cardinality of $R(x)$ and so on. We refer to the three sets as the colour classes of $x$ and the three cardinalities as the chromatic valencies or simply valencies of $x$. We sometimes write $R(x)$ to refer to the coloured graph induced by the set of vertices attached by red edges to $x$, instead of simply meaning the vertex-set of the graph; no problems arise.

We shall be concerned with monochromatic triangles throughout. Since no confusion will occur, it is convenient simply to say "triangle" when "monochromatic triangle" is meant. By a four-triangle colouring of $K_{17}$ we shall mean an edge-colouring of $K_{17}$ in which at most four triangles occur.

\section{Colouring $K_{6}$}

Among the 2-triangle 2-colourings of $K_{6}$ there are two special cases which we consider. 
Lemma 1. If a 2-colouring of $K_{6}$ includes exactly two triangles, and the triangles are disjoint, then they are the same colour.

Proof: Suppose the $K_{6}$ contains a red triangle $a b c$ and a blue triangle $x y z$. Since none of $a b x, a c x$ and $b c x$ can be a red triangle, at most one of $a x, b x$ and $c x$ can be red. Similarly at most one of $a y, b y$ and $c y$ can be red. So either $a x$ and $a y$ are both blue, or $b x$ and $b y$, or $c x$ and $c y$. In any case there is a blue triangle containing $x, y$ and some point other than $x$; this is a third triangle, which is impossible.

Lemma 2. Suppose a 2-colouring of $K_{6}$ contains exactly two triangles, and suppose they cover only four vertices $a, b, c, d$ between them; denote the other vertices by $z, w$. Then the edge $z w$ is the same colour as the edges of two triangles, and the graph of the edges of that colour is determined up to isomorphism: it is isomorphic to the one shown in Figure 1.

ProOF: The configuration spanned by $\{a, b, c, d\}$ must consist of a $K_{4}$ with five edges of one colour and one of another, so assume ad is blue and the other edges are red. The only triangles in the $K_{6}$ are $a b c$ and $b c d$.

Assume $z w$ is blue. Since $a z w$ is not a triangle, $a z$ and $a w$ cannot both be blue; assume $a z$ is red. Then $b z$ must be blue. Since $b z$ and $z w$ are blue, $b w$ must be red. Thus $c w$ must be blue, to avoid triangle $b c w$. What colour is $c z$ ? If it is red, then $a c z$ is a red triangle; if it is blue, then $c z w$ is a blue triangle-a contradiction. So $z w$ is red.

From now on we assume that $z w$ is red. We observe that not both $a w$ and $d w$ can be blue, since $a d w$ is not a triangle. Without loss of generality, assume $a w$ is red. Then both $b w$ and $c w$ are blue. Moreover $a z$ is blue, to avoid a red triangle $a w z$. So $d z$ is red (to avoid triangle $a d z$ ), and $d w$ is blue (to avoid triangle $d w z$ ). It is easy to see that the edges $b z$ and $c z$ must also be blue. We obtain the configuration of red edges in Figure 1 (any edge of $K_{6}$ not shown in the diagram is coloured blue). The graph is unique, except for the possibility of changing to an isomorphic version by relabelling among $\{a, b, c, d\}$ or $\{w, z\}$.

LEMMA 3. If a 2-colouring of $K_{6}$ contains exactly two triangles and between them they contain exactly five points, then the two triangles are of different colours.

Proof: Suppose not: say $a b c$ and ade are the triangles, and both are red. Then $b d, b e, c d$ and $c e$ must all be blue. If $f$ is the sixth point then at most one of $b f$ and $c f$ can be red; without loss of generality, say $b f$ is blue. Similarly either $d f$ or $e f$ is blue. In the first case $b d f$ is a blue triangle, and in the second case bef is blue; in either case we have an impossiblity. 


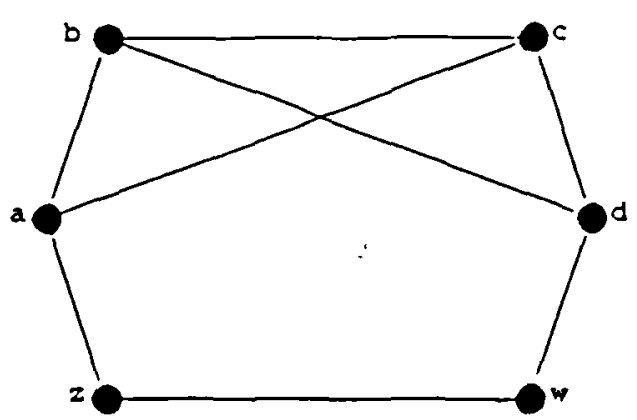

FIGURE 1

\section{Triangle-free vertices}

Suppose the vertex $x$ does not lie in any triangle in a 3-colouring of the edges of $K_{17}$. Such a point will be called triangle-free relative to the colouring. Then it is clear that $R(x)$ can contain no red edges, $B(x)$ no blue edges and $G(x)$ no green edges. If $R(x)$ has $r$ vertices, it will contain at least $f(r)$ triangles (where $f(r)$ was defined in Lemma 2) because it consists of a copy of $K_{r}$ coloured in blue and green. Such considerations tell us immediately that if $x$ is a triangle-free vertex in a 3-colouring which contains at most four triangles altogether, then $x$ can have no valency greater than 7 . We can also eliminate the possibility of valency 7 , but first we need a Lemma.

LEMMA 4. No 2-colouring of $K_{7}$ which contains exactly four triangles can contanin a monochromatic $K_{4}$.

Proof: Suppose $K_{\tau}$ is coloured in blue and green, and has a blue $K_{4}$ with vertices $x, y, z, w$. Consider the 2-colouring of $K_{6}$ obtained by deleting $w$. It must contain at least two triangles, one of which is $x y z$. Now these two triangles, together with $x y w, x z w$ and $y z w$, are all triangles of $K_{7}$ - at least five triangles.

TheOREM 1. Suppose $K_{17}$ is coloured with exactly four triangles, and $x$ lies on no triangle. Then $x$ can have no valency greater than 6 .

Proof: Assume the contrary: say $r(x) \geqslant 7$. Then $R(x)$ contains at least four triangles; from the data it can contain at most four, so $r(x)=7$; also, $b(x) \leqslant 5$ and $g(x) \leqslant 5$, to avoid further triangles.

Select $y$ and $z$ both in $B(x)$ or both in $G(X)$, such that $y z$ is red. Neither $y$ nor $z$ belong to triangles, since all four triangles are in $R(x)$. Suppose $M$ is a colour such that $m(y) \geqslant 6$ (there must be one, since the valencies of $y$ add to 16). Since $y$ lies on no triangle, $M(y)$ contains at least two triangles. Say $T_{y}$ is the union of the vertex-sets of two triangles in $M(y)$. Now $T_{y}$ has at least 4 elements, and $T_{y}$ lies in $M(y) \cap R(x)$. 
If $M=B$, then all edges joining members of $T_{y}$ must be green (they cannot be red, as that would put $x$ in a triangle; and if any were blue we would have a triangle containing $y$ ). Similarly $M=G$ gives a monochromatic $K_{4}$ in $R(x)$ but this would mean there are at least five triangles, by Lemma 4: a contradiction. So $M=R$.

Now $R(x)$ and $R(y)$ have at least four common elements, the members of $T_{y}$. Similarly $R(x)$ and $R(z)$ have at least four common elements. Therefore $R(y)$ and $R(z)$ have a common element, $w$ say, and $y z w$ is a red triangle: an impossibility.

\section{ON $x$-BASED COLOURINGS}

Since we shall always be considering one special case in this section, it is convenient to introduce a corresponding special definition. We define an $x$-based colouring of $K_{17}$ to be an edge-colouring in which there are precisely four triangles, and in which the particular vertex $x$ lies on no triangles and has valencies $r(x)=b(x)=6, g(x)=4$. We shall prove that no $x$-based colouring exists.

Consider an $x$-based colouring. Since $x$ is on no triangle, $R(x)$ is coloured entirely in green and blue; so it contains at least two triangles. Similarly $B(x)$ contains at least two triangles. As the total number of triangles is precisely four, we have the following.

LeMma 5. In an $x$-based colouring, each of $R(x)$ and $B(x)$ contains precisely two triangles and no triangle lies in $G(x)$ or has vertices in more than one of the sets $R(x), B(x)$ or $G(x)$.

Since $G(x)$ contains no red triangle and no green edge, it is easy to see that it either consists of a red path and a blue path, each of length 3 , or else it consists of a 4-cycle in one colour and two independent edges in the other. Therefore every vertex in $G^{\prime}(x)$ lies on edges of both colours. In symbols this can be expressed as follows:

LEMMA 6. In an $x$-based colouring, if $y$ belongs to $G(x)$, then

$$
|R(y) \cap G(x)| \neq 0, \quad|B(y) \cap G(x)| \neq 0
$$

in fact, one set will have size 1 and the other size 2 .

Lemma 7. In an $x$-based colouring, any $y$ in $G(x)$ satisfies $g(y) \leqslant 5$.

Proof: Suppose not. Since $y$ can be in no triangle, Theorem 1 says $g(y) \leqslant 6$; so $g(y)=6$ and $G(y)$ contains at least two triangles. Now $x \in G(y)$, so

$$
|G(y) \cap B(x)|+|G(y) \cap R(x)|=5 .
$$

If one of the two intersections has size 3 and the other has size 2, then we cannot come to two triangles-the larger intersection can contain only one, the smaller none, 
and there can be no other triangle in $G(y)$ (any such triangle would either contain $x$ or else contain elements of both $R(x)$ and $B(x)$, an impossibility). So one intersection has at least four elements. Say $|G(y) \cap B(x)| \geqslant 4$. Since all edges of $G(y) \cap B(x)$ must be red, we have a red $K_{4}$, which is made up of four red triangles. So $B(x)$ contains four triangles - too many.

LEMMA 8. In an $x$-based colouring, neither $B(x)$ nor $R(x)$ can contain two triangles which between them cover only four points.

Proof: Suppose the two triangles in $B(x)$ cover only four points. Then Lemma 2 applies. Suppose the triangles are red, and suppose the vertices are labelled as in Figure 1 and the red edges are as shown in Figure 1. From Lemma 5, neither $w$ nor $z$ can belong to any triangle, so by Theorem 1 none of their valencies is greater than 6 . Suppose $b(z)=6$ Then $B(z)$ contains at least two triangles. These triangles must lie entirely within $R(x)$, by Lemma 5 . So $B(z) \cap R(x)$ has four (or more) vertices, which must constitute a green $K_{4}$, giving too many triangles. So $b(z)<6$.

Next suppose $g(z)=6$. Then $G(z)$ contains two triangles. One is $b c d$, which is red. The other must lie entirely within $R(x)$, so it is not red. Therefore $G(z)$ is a two-coloured $K_{6}$ with two triangles of different colours. From Lemma 1 , it must contain a further triangle, so the $K_{17}$ contains a triangle which touches both $B(x)$ and $R(x)$ : an impossibility. So $g(z)<6$.

It follows that $r(z)=6$, and similarly $r(w)=6$. Now $R(z)$ must contain two triangles, and neither $w$ nor $a$ wil be in either of them. So $R(z) \cap R(x)$ has four elements. Similarly $R(w) \cap R(x)$ has four elements. Since $r(x)=6$, there must be at least two points in common to $R(z)$ and $R(x)$, and $z$ and $w$ lie in at least two red triangles: an impossibility.

(ii) Suppose now that the triangles in $B(x)$ are green. Again we assume that the points of $B(x)$ are labelled as in Figure 1, but we assume that the figure shows the green edges.

Let us label the members of $G(x)$ as $\{p, q, s, t\}$. None of these points lies in a triangle, so each has a maximum valency of 6 . From Lemma 7 , none has a green valency of 6 .

Suppose $r(p)=6$. Then $R(p)$ contains two triangles. As $p$ lies on a red edge in $G(x)$ (from Lemma 6), we cannot have $|R(p) \cap B(x)|=|R(p) \cap R(x)|=3$, so the two triangles cannot be one in $B(x)$ and one in $R(x): R(p)$ must have at least four vertices in common with one of the two sets $R(x)$ and $B(x)$. If $R(p) \cap B(x)$ had four vertices, they would span a green $K_{4}$, which is impossible. So $R(p) \cap R(x)$ has at least four elements, and contains all the vertices of the two triangles in $B(x)$.

If there were three points in $G(x)$ whose red valencies all equalled 6 , then no 
two of them could be joined by a red edge,so they would form a blue triangle, which is impossible. So $G(x)$ contains at most two vertices with red valency 6 . The same argument shows that there are at most two vertices with blue valency 6 . So there must be two of each kind. Without loss of generality we assume

$$
r(p)=r(q)=6, \quad b(s)=b(t)=6 ;
$$

necessarily both the other valencies of each vertex must equal 5 . Moreover $s$ and $t$ are both joined by blue edges to each of $a, b, c$ and $d$. Edge $p q$ must be blue and $s t$ must be red. To avoid triangles, $G(x)$ must consist of two paths of length 3 ; without loss of generality, assume $p s$ and $q t$ are blue, and $q s$ and $p t$ are red.

Two blue edges go from $p$ to $G(x)$, and at most two to $R(x)$. So $B(x) \cap B(p)$ has at least one member. It cannot be $a, b, c$ or $d$, because that would result in a blue triangle with $p s$. So either $p z$ or $p w$ is blue - but not both, for either $z$ or $w$ must also be connected to $q$ by a blue edge. Let us assume that $p z$ is blue. Then $|B(p) \cap R(x)|$ must equal 2 , and $|R(p) \cap R(x)|=4$.

Now consider $z$. Just as in part (i) we see that $b(z)<6$. If $g(z)=6$ then $G(z)$ contains two triangles; since $G(z) \cap B(x)=\{a, w\}$ which contains no triangle, and $G(z) \cap R(x)$ has at most three elements, this is impossible. So $g(z)<6$, and $r(z)=6$, $b(z)=g(z)=5$.

$R(z)$ contains two triangles. One is $b c d$; the other must lie entirely in $R(x)$. So $R(z) \cap R(x)$ has three elements, all of which lie in $R(p)$.

Where are the five elements of $B(z)$ ? No member of $B(x)$ can belong to it, nor can $q$ or $s$, and three of the members of $R(p) \cap R(x)$ are excluded. Also the elements of $B(p) \cap R(x)$ cannot be included, as they would form a blue triangle with $z p$. Only four possible vertices remain - a contradiction.

TheOREM 2. No $x$-based colouring of $K_{17}$ exists.

Proof: We first show that, in an $x$-based colouring, no member of $G(x)$ has $\{6,6,4\}$ as its set of valencies.

Suppose $y$ were such a vertex. By Lemma 7 and Theorem 1 we have $r(y)=b(y)=$ 6 and $g(y)=4$. So from Lemma 6 either

$$
|B(y) \cap G(x)|=2 \text { or }|R(y) \cap G(x)|=2 .
$$

We assume $|B(y) \cap G(x)|=2$ and obtain a contradiction; by symmetry, $|R(y) \cap G(x)|=$ 2 is impossible also.

Since $b(y)=6$ we have

$$
|B(y) \cap\{R(x) \cup B(x)\}|=4,
$$


so

$$
|B(y) \cap R(x)|+|B(y) \cap B(x)|=4 \text {. }
$$

If $B(y)$ intersects $R(x)$ in four elements, all members in that set of four elements must be joined by green edges, and we have a monochromatic $K_{4}$ in $R(x)$, an impossibility. Now $B(y)$ must contain two triangles, which must each lie entirely within $R(x)$ or entirely within $B(x)$, so the cases where one intersection has three members and the other one, or both have two, are easily eliminated. Therefore $|B(y) \cap B(x)|=4$.

Now suppose $a, b, c, d$ are the vertices of $B(y)$ which lie in $B(x)$. The other two members of $B(y)$ lie in $G(x)$. Since those two lie in no triangle, the vertices $a, b$, $c, d$ must form two triangles. Therefore $B(x)$ contains two triangles which together cover only four points, in contradiction of Lemma 8.

We can now assume that all members of $G(x)$ have valencies of $\{6,5,5\}$, where the green valency is 5 . Let $y$ be such a vertex; assume $r(y)=5$ and $b(y)=6$.

Since $y$ is on no triangle, $B(y)$ contains two triangles. As $B(y)$ contains at least one member of $G(x)$, either $|B(y) \cap R(x)| \geqslant 4$ or $|B(y) \cap B(x)| \geqslant 4$. In the former case $R(x)$ contains a green $K_{4}$, which is impossible. In the latter, if $|B(y) \cap B(x)|=4$ we obtain a contradiction via Lemma 7 . So $B(y) \cap B(x)$ has 5 elements, and necessarily $|B(y) \cap G(x)|=1$ and $|R(y) \cap G(x)|=2$ (using Lemma 4). Since $R(x) \cap G(y)$ cannot have four members, necessarily $|R(x) \cap R(y)| \geqslant 3$.

So a member $y$ of $G(x)$ must fit one of the following two descriptions:

(i) $y$ lies on one blue edge in $G(x)$, and

$$
|B(y) \cap B(x)|=5, \quad|R(y) \cap R(x)|=3 ;
$$

(ii) $y$ lies on two blue edges in $G(x)$, and

$$
|B(y) \cap B(x)|=3, \quad|R(y) \cap R(x)|=5 .
$$

Suppose $y$ lies on one blue edge in $G(x)$, the edge $y z$. Since $B(x)$ has only six elements, $B(y) \cap B(z)$ is non-empty, and $y z$ lies in a blue triangle. But this is impossible. Therefore $G(x)$ consists of a blue four-cycle and two independent red edges. But by the same argument the endpoints of the red edges must lie in a triangle: a contradiction. So no $x$-based colouring exists.

\section{Restrictions ON THE LARGE COLOUR CLASS}

From now on we can assume that any triangle-free vertex has valencies $\{6,5,5\}$. The colour in which the triangle-free vertex has valencey 6 will be called the large colour class of the vertex.

In this section we assume that $x$ is a triangle-free point in a 4-triangle colouring of $K_{17}$ whose large colour class is $R(x)$, so $r(x)=6$ and $b(x)=g(x)=5$. We prove that $R(x)$ cannot contain more than two triangles. 
LEMMA 9. $R(x)$ cannot contain a monochromatic $K_{4}$.

Proof: Suppose $R(x)$ contains a blue $K_{4}$. Consider any vertex $y$ in $B(x)$. Either $r(y)=6$, in which case $R(y)$ must contain two triangles, so $y$ is joined to the points of the $K_{4}$ by red edges, and we call $y$ a red point; or $g(y)=6$, the joins are all green, and we call $y$ a green point.

As $b(x)=5, B(x)$ either contains three red points or three green points. Suppose there are three red points. If any edge joining two of them is red, we get four red triangles formed by them and the vertices of the $K_{4}$. But no edge in $B(x)$ is blue. So the three edges are green and we have a green triangle, giving at least five triangles in total.

Although the above Lemma is written in terms of $R(x)$, it is clear that any monochromatic $K_{4}$ in a 4-triangle colouring would lie within $R(x)$, so no colouring contains a monochromatic $K_{4}$.

Theorem 3. $R(x)$ contains at most two triangles.

Proof: Suppose $R(x)$ contains three triangles; write $S$ for the set of vertices of those triangles. We first show that there must be another triangle $T$ which contains at most one vertex of $R(x)$. For, if not, any set of two triangles must contain four members of $R(x)$. Consider any triangle-free vertex $y$. If $g(y)=6$, then $G(y)$ contains two triangles and $|G(y) \cap R(x)| \geqslant 4$; we must have a blue $K_{4}$ in $R(x)$, which is impossible. Similarly $b(y) \neq 6$. So $r(y)=6$, and $|R(x) \cap R(y)| \geqslant 4$. This implies that for any two triangle-free vertices $y$ and $z, R(y)$ and $R(z)$ have non-empty intersection, and consequently $y z$ cannot be red. But there are at least eight triangle-free vertices, and we cannot colour the $K_{8}$ spanned by them in blue and green without a triangle. So the situation is impossible.

Now assume there is a fouth triangle $T$ with at most one vertex in $R(x)$. (Observe that if $T$ does have a vertex in $R(x)$, that vertex must be in $S$.) We distinguish two cases; $T$ is red, or $T$ is blue. (The proof when $T$ is green is the same as for $T$ blue.)

First, suppose $T$ is blue. Since $T \cap R(x)$ and $T \cap B(x)$ can have at most one element each, there is at least one vertex of $T$ in $G(x)$. If there is only one, so that $T$ consists of vertices $t_{r}, t_{b}$ and $t_{g}$ in $R(x), B(x)$ and $G(x)$ respectively, consider the two vertices $y$ and $z$ in $G(x)$ which are joined to $t_{g}$ by blue edges. Both are triangle-free. The edge $y z$ is red. Neither $t_{r}$ nor $t_{b}$ can belong to $B(y)$, so $T$ does not lie in $B(y), R(y)$ or $G(y)$. So, if any of those three sets has six elements, that set must contain two triangles in $R(x)$, so it contains at least four elements of $R(x)$. It follows that the six-element set must be $R(y)$, in order to avoid a monochromatic $K_{4}$. Similarly $r(z)=6$ and $|R(x) \cap R(z)| \geqslant 4$. So there is a common element of $R(y)$ and $R(z)$ and it forms a red triangle with $y z$. 
On the other hand, if $T \cap G(x)$ has two elements, $t$ and $w$ say, consider the two further points $y$ and $z$ in $G(x)$ which are joined to $t$ and $w$ respectively by blue edges. If $M(y)$ is the six-element set joined to $y$ by one colour, then $M \neq B$; if $M=G$ then $G(y) \cap R(x)$ has four members, a blue $K_{4}$; so we have $M(y)=R(y)$ and $M(z)=R(z)$. Since $y z$ is necessarily red, we again derive a contradiction.

Now suppose $T$ lies entirely within $G(x)$. Select any member $z$ of $B(x)$ and suppose $M$ is the colour such that $m(z)=6$. If $T$ is in $M(z)$ then $M(z)$ must contain the three members of $T$ and also the three members of $R(z)$ which belong to some triangle. If $M=B$, then $M(x)$ also contains $x$; otherwise $M(x)$ contains two members of $B(x)$, since $B(x)$ consists of red and green 5 -cycles. So $M(z)$ cannot contain $T$. We then deduce that $M(z)=R(z)$, and that $R(z)$ contains four of the six members of $R(x)$. The existence of red edges in $B(x)$ implies a red triangle containing two members of $B(x)$, which is impossible.

We now assume $T$ is red. If $T$ lies entirely within $G(x)$ the above proof remains valid, since we did not use the colour of $T$; and the proof when $T$ lies in $B(x)$ is analogous. So we can assume that $T$ lies in two of the sets. First assume that $T$ has no point in $R(x)$; without loss of generality, say $|T \cap G(x)|=2$ and $|T \cap B(x)|=1$. Say $z$ is the point of $T$ which is in $B(x)$, and let $w$ and $t$ be the points of $B(x)$ which are connected to $z$ by green edges.

What is the 6-element set $M(w)$ ? If $M(w)=B(w)$ then $B(w) \cap R(w)$ has four elements, which is impossible. If $M(w)=G(w)$ then $G(w)$ must contain three vertices of a triangle from $R(x)$, the three points of $T$ and at least one further point of $B(x)$, which gives too many points, or else $G(w) \cap R(x)$ has four points, which is impossible. So $M(w)=R(w)$, and $|R(w) \cap R(x)| \geqslant 4$. But $|R(t) \cap R(x)| \geqslant 4$ also, so $R(t)$ and $R(w)$ have a common element, and $t w$ is a red edge, so $t w$ lies in a red triangle - a contradiction.

So finally $T$ must be red and must have a vertex $t$ in $R(x)$; as we have noted, $t$ must be a vertex of one of the triangles entirely within $R(x)$. We can also assume that $T$ has one or two vertices in $G(x)$ (if they both lie in $B(x)$, we simply reverse blue and green throughout).

Say $T \cap G(x)=\{y, z\}$. Let us label the other vertices in $G(x)$ as $u, v, w$ where $(u, y, z, v, w)$ is a red 5 -cycle and the other five edges are blue. If $M$ is the colour such that $m(u)=6$, then $M(u)$ does not contain $T$ (since $T$ is joined to $u$ by both red and blue edges), so $M(u)$ contains at least four elements of $R(x)$ and $M=R$. Simularly $r(v)=6$ and $R(v)$ contains at least four elements of $R(x)$. As $R(u)$ and $R(v)$ each contain two elements of $G(x)$, it follows that

$$
|R(u) \cap R(x)|=|R(v) \cap R(x)|=4 .
$$

Moreover, both $R(u)$ and $R(v)$ contain two triangles in $R(x)$. Since neither contains 
$t$, they do not contain the triangle in $R(x)$ which contains $t$. So they contain the same two triangles, and $R(u) \cap R(x)=R(v) \cap R(x)$.

Now $B(u)$ does not contain any member of $B(v)$ (because $u$ and $v$ do not lie in a blue triangle) and the only member of $B(u)$ in $R(v)$ is $z$. (The other five members of $R(v)$ are accounted for $-w$ and four members of $R(x)$-and all lie in $R(u)$.) So $|B(u) \cap G(v)|=3$. Those three points must form a red triangle which is not one of the four triangles we already know: a contradiction.

Lastly, say $T \cap G(x)=\{y\}, T \cap B(x)=\{z\}$. Let $u$ and $v$ be the two vertices in $B(x)$ joined to $z$ by red edges. The edge $u v$ is green. Just as before we see that $r(u)=r(v)=6$ and that $R(u)$ and $R(v)$ contain the same four elements of $R(x)$. They also each contain $z$, and $R(u) \cap G(v)$ and $G(u) \cap R(v)$ each have one element, which is in $B(x)$. So again $|B(u) \cap G(v)|=3$ yielding a new red triangle, a contradiction.

\section{The MaIN Result}

Suppose $x$ is a triangle-free vertex in a four-triangle colouring of $K_{17}$. From the previous sections, $x$ has valencies $\{6,5,5\}$; and if $m(x)=6$ then $M(x)$ contains exactly two triangles. If those triangles are $T_{1}$ and $T_{2}$, define $f(x)$ to equal $\left\{T_{1}, T_{2}\right\}$. Then $f$ is a well-defined map from the triangle-free points of $K_{17}$ to the unordered pairs of triangles in the colouring.

Lemma 10. Suppose $f(x)=f(y)$. Then the large colour classes of $x$ and $y$ are the same colour.

Proof: Suppose $f(x)=f(y)=\left\{T_{1}, T_{2}\right\}$. Then the large colour classes of $x$ and $y$ both contain the four or more members of $T_{1} \cup T_{2}$. If the two large classes are of different colours, then all the edges between those vertices must be the same colour: for example, if $R(x) \cap B(y)$ has four elements, then all the edges joining the four points must be green. But a monochromatic $K_{4}$ is impossible, by Lemma 9 .

LEMma 11. If $T_{1}$ and $T_{2}$ are two triangles with 0 or 1 common points, then there cannot exist distinct triangle-free points $x$ and $y$ with $f(x)=f(y)=\left\{T_{1}, T_{2}\right\}$.

Proof: Suppose $f(x)=f(y)=\left\{T_{1}, T_{2}\right\}$. Suppose also that the large colour class of $x$ is $R(x)$. Then the large colour class of $y$ is $R(y)$, and $R(x) \cap R(y)$ has at least five elements. Clearly, $x y$ is not red; suppose it is blue. Then $B(x) \cap B(y)$ has no elements; since $r(x)=6, R(x) \cap B(y)$ can have at most one element; so $G(x) \cap B(y)$ has at least three elements.

If $T_{1}$ and $T_{2}$ are disjoint then $|R(x) \cap R(y)|=6, R(x) \cap B(y)$ is empty, and $|G(x) \cap B(y)|=4$. Then $G(x) \cap B(y)$ is a blue $K_{4}$, which is impossible. So $T_{1}$ and $T_{2}$ cannot be disjoint. 
If $T_{1}$ and $T_{2}$ have one common element, write $T_{3}$ for $G(x) \cap B(y)$ and $T_{4}$ for $B(x) \cap G(y)$. Both $T_{3}$ and $T_{4}$ must be red triangles, and each is disjoint from $T_{1}$ and $T_{2}$. Neither $T_{1}$ nor $T_{2}$ is red. It follows from Lemma 1 that $\left\{T_{1}, T_{3}\right\},\left\{T_{1}, T_{4}\right\}$, $\left\{T_{2}, T_{3}\right\}$ and $\left\{T_{2}, T_{4}\right\}$ can never be values of $f(z)$ for any triangle-free vertex $z$. Moreover, from the first part of this proof, $\left\{T_{3}, T_{4}\right\}$ cannot equal $f(z)$ for more than one value of $z$. Since $T_{1} \cup T_{2} \cup T_{3} \cup T_{4}$ contains eleven points, there must be five different points with $f=\left\{T_{1}, T_{2}\right\}$. Call them $x, y_{1}, y_{2}, y_{3}, y_{4}$. Now the intersections of $G(x)$ and $B\left(y_{1}\right), B\left(y_{2}\right), B\left(y_{3}\right)$ and $B\left(y_{4}\right)$ are all triangles; since $B(x)$ can contain only one triangle, these intersections must be equal. So $B\left(y_{i}\right) \cap B\left(y_{j}\right)$ is non-empty for all $i$ and $j$, and $y_{i} y_{j}$ cannot be blue. But it also cannot be red: $R\left(y_{i}\right) \cap R\left(y_{j}\right)$ contains $T_{1} \cup T_{2}$. So we have a green $K_{4}$, which is impossible.

LEMмa 12. Suppose $T_{3}$ and $T_{3}$ are triangles with two common points. Then there can be no more that two vertices $x, y$ such that $f(x)=f(y)=\left\{T_{1}, T_{2}\right\}$.

Proof: Suppose $T_{1}=a b c$ and $T_{2}=b c d$. Without loss of generality we assume that two triangles are blue and that they lie in $R(x)$, which is the large colour class of $x$. By Lemma $8, R(y)$ is the large colour class of $y$. Clearly $x y$ cannot be red.

First assume $x y$ is blue. There are two vertices in $R(x)$ but not in $T_{1} \cup T_{2}$. Call them $u$ and $v$. By Lemma 2, $u v$ is blue. So $u y$ and $v y$ cannot both be blue. Therefore, $B(y) \cap R(x)$ has at most one member. But $B(y) \cap B(x)$ must be empty. Therefore $B(y) \cap G(x)$ has three elements which must constitute a red triangle $T_{3}$. Similarly $G(y) \cap B(x)$ must constitute a red triangle $T_{4}$. As $T_{1}$ and $T_{2}$ are disjoint and have different colours, no triangle-free point $z$ has $f(z)=\left\{T_{1}, T_{2}\right\}$. Similarly $\left\{T_{1}, T_{4}\right\},\left\{T_{2}, T_{3}\right\}$ and $\left\{T_{2}, T_{4}\right\}$ are impossible. From Lemma 10, at most one point can have $f(z)=\left\{T_{3}, T_{4}\right\}$. Since $\left|T_{1} \cup T_{2} \cup T_{3} \cup T_{4}\right|=10$, there must be six triangle-free points $x_{1}, x_{2}, x_{3}, x_{4}, x_{5}, x_{6}$ such that $f\left(x_{i}\right)=\left\{T_{1}, T_{2}\right\}$ and $R\left(x_{i}\right) \cap R\left(x_{j}\right)$ contains $T_{2} \cup T_{2}$. All edges $x_{i} x_{j}$ must be blue or green, so we get at least two more triangles: an impossibility.

So $x y$ is green. If there is a third point $z$ with $f(z)=\left\{T_{1}, T_{2}\right\}$ then $x y z$ is a green triangle, which is impossible, proving the Lemma.

One easy observation which is now available to us is that at least four triangles must appear in any 3 -colouring of the edges of $K_{17}$ : if there were only three triangles, then there could only be three possible values of the function $f$, and consequently there would be at most six triangle-free points; not enough points in total. (Although most. of the subcases have been such as to guarantee four triangles, we had not outlawed the three-triangle possibility until now.) The union of the four triangles must contain between five and twelve points (four points would mean a monochromatic $K_{4}$, banned by Lemma 9 ; fewer points could not give four triangles). Given a four-triangle colouring 
of $K_{17}$ we write $T_{1}, T_{2}, T_{3}, T_{4}$ for the four triangles and $T$ for their union (considered as a set of points); we show that the values of $|T|$ from 5 to 12 are impossible.

It will be useful to establish some notation. The set of triangle-free points is labelled $F$; it has $17-|T|$ elements. Also we call a pair of triangles $\left\{T_{1}, T_{2}\right\}$ rich if there are two points $x$ and $y$ in $F$ such that $f(x)=f(y)=\left\{T_{1}, T_{2}\right\}$. A rich pair spans four points and consequently both triangles are the same colour.

THEOREM 4. $|T| \neq 12$.

Proof: Suppose $|T|=12$. There are four disjoint triangles. Five of the six possible pairs are images under $f$, so there is one triangle, $T_{1}$ say, such that $\left\{T_{1}, T_{2}\right\}$, $\left\{T_{1}, T_{3}\right\}$ and $\left\{T_{1}, T_{4}\right\}$ are all images. Consequently the triangles are all the same colour, blue say. Suppose $f(x)=\left\{T_{1}, T_{2}\right\}$, and say $T_{1}$ and $T_{2}$ belong to $R(x)$. Then $B(x)$ contains at most two points, $y$ and $z$ say, from the other two triangles, so it contains three triangle-free points $a, b, c$. Since $B(x)$ contains no triangle and no blue edge, one of these three must be connected to the other by edges of different colours, not blue-say $a b$ is red and $a c$ is green. Let $M(a)$ be the large colour class of $a$. Then $M(a) \neq R(a)$ and $M(a) \neq G(a)$, since $M(a)$ is the union of two disjoint triangles and thus it may not contain a triangle-free point such as $b$ or $c$. So $M(a)=B(a)$; but then $B(a)$ contains blue triangles, which is impossible.

Theorem 5. $|T| \neq 11$.

Proof: Suppose $|T|=11$. Say $T_{1} \cap T_{2}$ has one point; $T_{3}$ and $T_{4}$, the other triangles, are disjoint from $T_{1} \cup T_{2}$ and from each other.

There are six triangle-free points; from Lemma 10 there are no rich pairs; so every pair of triangles must appear as one image under $f$. Since $\left\{T_{1}, T_{2}\right\},\left\{T_{2}, T_{4}\right\}$ and $\left\{T_{3}, T_{4}\right\}$ are disjoint pairs, all four triangles must have the same colour.

But suppose $f(x)=\left\{T_{1}, T_{2}\right\}$, which must occur for some $x$. Say $T_{1} \cup T_{2} \subseteq R(x)$. Since $T_{1}$ and $T_{2}$ are the same colour, this contradicts Lemma 3 .

THEOREM 6. $|T| \neq 10$.

Proof: Suppose $|T|=10$. Then there must be a rich pair of triangles, $T_{1}$ and $T_{2}$. Those triangles must be the same colour. To attain 10 points, $T_{3}$ and $T_{4}$ must be disjoint from $T_{1} \cup T_{2}$ and from each other; just as in the proof of Theorem 4, all must be the same colour-say blue. Again as in Theorem 4 we can find a triangle-free point in $B(x)$ which is joined to other triangle-free points by a red edge and a green edge, and we have a contradiction. (Since $x y$ is green, $a \neq y$, so $f(a)$ consists of two disjoint triangles.)

ThEOREM 7. $|T| \neq 9$. 
Proof: If $|T|=9$ then $F$ has 8 members so there are at least two rich pairs. Say $\left\{T_{1}, T_{2}\right\}$ is rich, and $T_{1} \cup T_{2}$ contains four points. Without loss of generality, the other rich pair is $\left\{T_{1}, T_{3}\right\}$ or $\left\{T_{3}, T_{4}\right\}$. In the former case, $T_{1} \cup T_{2} \cup T_{3}$ cannot contain more than five points; in the latter $T_{3} \cup T_{4}$ has only four. In either case $|T| \leqslant 8$. So $|T| \neq 9$.

THEOREM 8. $|T| \neq 8$.

Proof: Suppose $|T|=8$. There are nine triangle-free points, so there must be at least three rich pairs. Thus there must be some set of triangles $T_{1}, T_{2}, T_{3}$ such that $\left\{T_{1}, T_{2}\right\}$ and $\left\{T_{1}, T_{3}\right\}$ are rich. Since $T_{1} \cup T_{2}$ contains only four points, and $T_{3}$ can contain one point outside of $T_{1}$, therefore $T_{4}$ must be disjoint from the others to yield $|T|=8$, and $\left\{T_{2}, T_{3}\right\}$ must be the other rich pair; if there were more than three rich pairs then not even eight points are possible. It is easy to see that the only possible configuration is that shown in Figure 2, where the three triangles in rich pairs have a common edge: $T_{1}=a b d, T_{2}=a b c, T_{3}=a b e, T_{4}=u v w$ say.
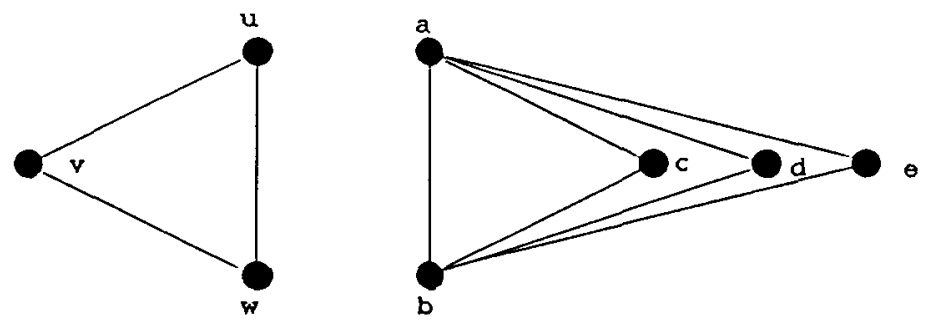

FIGURE 2

Even allowing every rich pair to be the image of two points under $f$, there must be a point whose image is each non-rich pair: write

$$
f(x)=\left\{T_{1}, T_{4}\right\}, \quad f(y)=\left\{T_{2}, T_{4}\right\}, \quad f(z)=\left\{T_{3}, T_{4}\right\}
$$

Now Lemma 1 tells us that all four triangles are the same colour-blue say. Let $R(x)$ be the large colour class of $x: R(x)=T_{1} \cup T_{4}$. So all the edges joining $T_{1}$ to $T_{4}$ must be blue or green, and at least two of the edges $a u, a v, a w$ must be green (or else we would have a blue triangle). Since the large colour class of $y$ is $T_{2} \cup T_{4}$, it must also be $R(y)$ (since it contains $a u$, av and $a w$ ) and the large colour class of $z$ is $R(z)$.

Now consider the edges joining $c, d, e$ to $u, v, w$. To avoid a blue triangle involving $c$ and an edge of $u v w$, at least two of $c u, c v$ and $c w$ must be green, and 
so also must two of $d u, d v$ and $d w$. If $c d$ is green we have a green triangle. But this means $c d$ must be red; and similarly for $c e$ and $d e$. So we have a red triangle - a contradiction.

TheOREM $9 .|T|>7$.

Proof: If $|T| \leqslant 7$ we must have four or more rich pairs, and if $|T| \leqslant 6$ then there must be at least five rich pairs. We first show that the case of four rich pairs is impossible. If there are exactly four rich pairs, then without loss of generality we can take them to be either $\left\{T_{1}, T_{2}\right\},\left\{T_{1}, T_{3}\right\},\left\{T_{1}, T_{4}\right\}$ and $\left\{T_{2}, T_{3}\right\}$ or $\left\{T_{1}, T_{2}\right\},\left\{T_{2}, T_{3}\right\}$, $\left\{T_{2}, T_{4}\right\}$ and $\left\{T_{4}, T_{1}\right\}$. In either case, $T_{1} \cup T_{2}$ can contain only four points, $T_{3}$ can contain at most one not in $T_{1} \cup T_{2}$, and $T_{4}$ at most one not in $T_{1} \cup T_{2} \cup T_{3}$. This gives only six points, a contradiction. So at least five rich pairs are needed.

Suppose there are five rich pairs; all the possibilities except perhaps $\left\{T_{3}, T_{4}\right\}$. Without loss of generality, $T_{1}=a b c$ and $T_{2}=a b d$; the only triangles to form a rich pair with both of these are $T_{3}=b c d$ or acd or abe. The first two possibilities give monochromatic $K_{4}$, which was elininated by Lemma 8 . The other case can be extended by $T_{4}=a b f$. So there is no case of five rich pairs, and only one of six, to be eliminated.

In the latter case, suppose the four triangles are all blue. Since $F$ has 11 elements, and since none of them have large colour class blue, these must be either at least 6 with large class red or at least 6 with large colour class green. Suppose 6 have large colour class red. If $x$ and $y$ are both in that set of six, then $a \in R(x) \cap R(y)$, so $x y$ is not red. We have a $K_{6}$ coloured in blue and green. But this must contain two triangles a contradiction.

In view of the remarks before Theorem 4, we have established Proposition 3.

\section{REFERENCES}

[1] A.W. Goodman, 'On Sets of acquaintances and strangers at any party', Amer. Math. Monthly 66 (1959), 778-783.

[2] A.W. Goodman, 'Triangles in a complete chromatic graph with three colors', Discrete Math. 57 (1985), 225-235.

[3] R. E. Greenwood and A. M. Gleason, 'Combinatorial relations and chromatic graphs', Canad. J. Math. $T$ (1955), 1-7.

[4] F. Harary, 'The two-triangle case of the acquaintance graph', Math. Mag. 45 (1972), $130-135$.

[5] F. Harary and G. Prins, 'Generalized Ramsey theory for graphs IV: The Ramsey multiplicity of a graph', Networks 4 (1974), 163-173.

[6] W.D. Wallis, 'The number of monochromatic triangles in edge-colourings of a complete graph', J. Combin. Inform. System. Sci. 1 (1976), 17-20.

[7] W.D. Wallis, A.P. Street and J.S. Wallis, Combinatorics: Room Squares, Sum-free Sets, Hadamard Matrices, Lecture Notes in Math. 292 (Springer-Verlag, Heidelberg, 1972). 
Center of Advanced Study in Mathematics University of Bombay

Bombay 400098

India
Department of Computer Science and Department of Mathematics

Southern Illinois University at Carbondale Carbondale, Illinois 62901

United States of America 